\title{
Dimethylcarbene vs. Direct Propene Formation in Dimethylketene Photodissociation
}

\section{Supporting Information- J. Phys. Chem. A.}

\author{
Sagnik Datta and H. Floyd Davis* \\ Department of Chemistry and Chemical Biology \\ Cornell University \\ Ithaca, NY 14853-1301
}

The photochemistry of gaseous dimethylketene was studied using photofragment translational energy spectroscopy at four excitation wavelengths. The absorption spectrum of dimethylketene in the $300-500$ $\mathrm{nm}$ wavelength range consists of a broad but weak continuum that is similar to, but slightly redshifted relative to that of ketene and methylketene (Fig. S1). Laser pulses at $355.1 \mathrm{~nm}(\sim 30 \mathrm{~mJ}, 7 \mathrm{~ns}, 30 \mathrm{~Hz})$ were produced by frequency tripling the unseeded output $\left(\sim 1 \mathrm{~cm}^{-1}\right)$ from a Continuum PL9030 Nd:YAG laser. Pulses $\left(\sim 10 \mathrm{~mJ}, \sim 0.2 \mathrm{~cm}^{-1}\right)$ at $320.0 \mathrm{~nm}, 340.0 \mathrm{~nm}$, and $371.0 \mathrm{~nm}$ were obtained using a Lambda-Physik Scanmate 2 dye laser pumped by the $532.2 \mathrm{~nm}$ output from an injection-seeded Continuum Precision 9030 $\mathrm{Nd}$ :YAG laser. For 320.0 and $340.0 \mathrm{~nm}$ generation, the visible output from the dye laser was frequency-doubled in a $\mathrm{BBO}$ crystal, whereas the 371.0 pulses were produced by mixing the visible dye laser output with the $1064.4 \mathrm{~nm}$ output of the Nd:YAG laser. To double the signal to noise ratio at $320.0,340.0$ and $371.0 \mathrm{~nm}$, the laser beam was double-passed through the interaction region using a retroreflecting dielectric mirror located in the vacuum chamber. In the $355.1 \mathrm{~nm}$ experiments, the laser beam was focused with a $750 \mathrm{~mm}$ focal

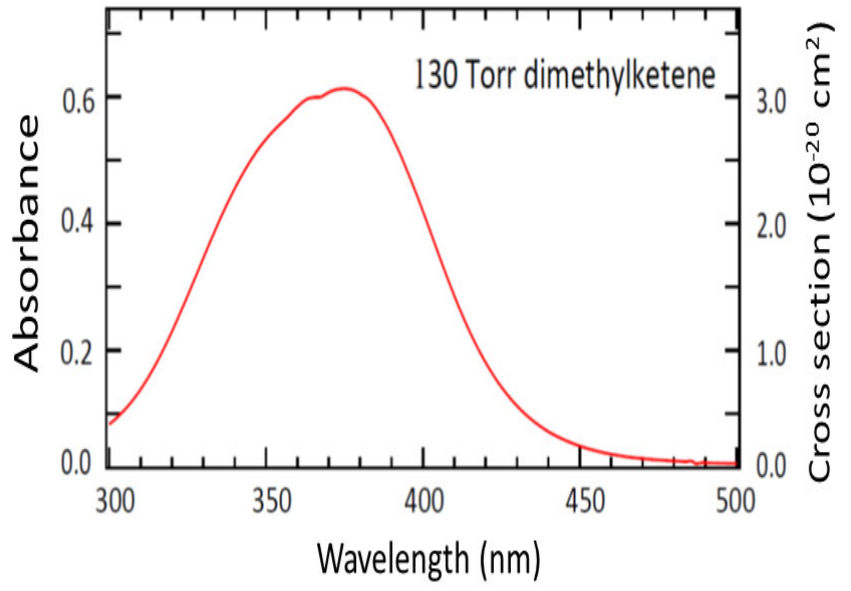

Fig. S1: UV-visible absorption spectrum for gaseous dimethylketene at 130 Torr in a $10 \mathrm{~cm}$ long cell. The calculated absorption cross sections are also indicated. length lens to a diameter of approximately 3 $\mathrm{mm}$ at the interaction region. The laser beam at the other wavelengths was unfocussed. 
The dimethylketene was prepared by flash vacuum pyrolysis of isobutyric anhydride (Aldrich). The precursor was passed at low pressure through a 1 " diameter stainless steel tube heated to $\sim 600^{\circ} \mathrm{C}$; the product mixture then flowed through a U-tube cooled to $-35^{\circ} \mathrm{C}$ to trap byproducts. The dimethylketene was subsequently trapped at $-195^{\circ} \mathrm{C}$ and then purified by vacuum distillation from a trap cooled to $-35^{\circ} \mathrm{C}$, with the middle fraction collected at $-195^{\circ} \mathrm{C}$. The dimerization process appears to be catalyzed by the presence of isobutyric acid impurities. However, we found that carefully purified liquid dimethylketene undergoes only slow dimerization at $-35^{\circ} \mathrm{C}$, over a span of many hours. The sample was redistilled or replaced each day to eliminate the accumulated dimeric impurities.

The molecular beam was produced by bubbling helium at 2 PSIG through the yellow liquid dimethylketene sample held at $-35^{\circ} \mathrm{C}$ in a thermostatically controlled bath. A pulsed molecular beam $(\sim 1 \%$ in $\mathrm{He}$ ) was produced using a room temperature home-built twin-piezo actuated pulsed valve and then collimated by two conical skimmers and crossed at a $90^{\circ}$ angle with the excitation laser beam $(\sim 2 \mathrm{~mm}$ diameter). ${ }^{1}$ To avoid photolysis of van der Waals dimers and larger clusters, the timing of the laser was adjusted to coincide with the arrival of the earliest and warmest part of the molecular beam pulse. While the $\mathrm{m} / \mathrm{e}=41$ and 40 signals were not appreciably affected by the photodissociation of van der Waals clusters, a very strong additional component with low translational energies was observed at $\mathrm{m} / \mathrm{e}=42$ and attributed to photodissociation of van der Waals clusters. Similar erroneous signals were observed at the parent dimethylketene mass $(\mathrm{m} / \mathrm{e}=70)$.

During the experiment, the pressure in the interaction region was held below $1 \times 10^{-6}$ Torr, thereby ensuring that photodissociation products drift collisionfree $15.2 \mathrm{~cm}$ to a liquid nitrogen cooled triplydifferentially pumped UHV detector $\left(\mathrm{P}<1 \times 10^{-10}\right.$ Torr during the experiment). There, the neutral products were photoionized at $9.9 \mathrm{eV}$ or $8.8 \mathrm{eV}$ using a highintensity pulsed vacuum ultraviolet (VUV) laser, described in detail elsewhere. ${ }^{2}$ The delay time of the VUV laser was scanned in $2 \mu$ s steps to obtain product TOF distributions. The molecular beam source is

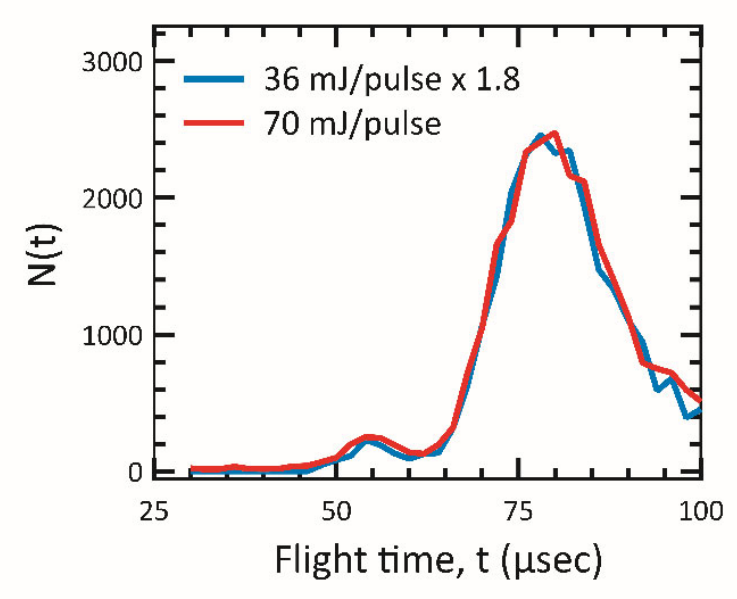

Fig. S2: TOF spectra for products from 355.1 $\mathrm{nm}$ photodissociation of $\mathrm{m} / \mathrm{e}=42$ dimethylketene at 10 degrees using $9.9 \mathrm{eV}$ photoionization at two laser pulse energies: The data recorded at the lower pulse energy (blue) was multiplied by a factor of 1.8 . 


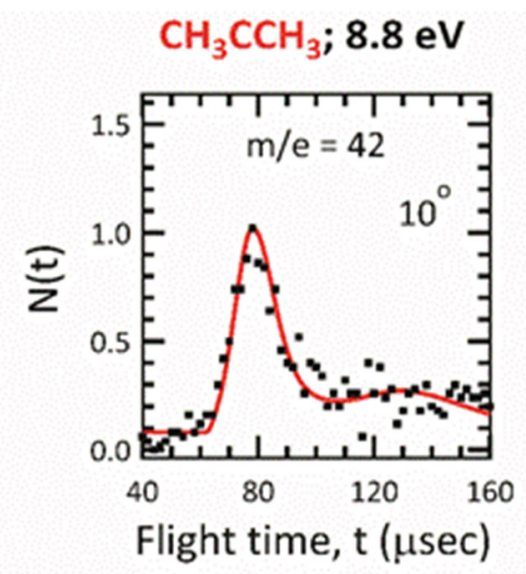

Fig. S3: TOF spectra for products from 355.1 $\mathrm{nm}$ photodissociation of dimethylketene at 10 degrees using $8.8 \mathrm{eV}$ photoionization: black dots are experimental data; red line is calculated TOF for $\mathrm{CH}_{3} \mathrm{CCH}_{3}+\mathrm{CO}$ channel. The relevant $P(E)$ is depicted in Fig. 2 of the main article. rotatable relative to the fixed detector; in each experiment the product arrival flight times were typically measured at several different angles. ${ }^{3}$

In Fig. S2, the TOF spectra are shown for $\mathrm{m} / \mathrm{e}=42$ for $355.1 \mathrm{~nm}$ photodissociation of dimethylketene at 10 degrees using $9.9 \mathrm{eV}$ photoionization at two laser pulse energies. For comparison purposes, the data at the lower pulse energy (blue) was multiplied by a factor of 1.8. The shapes of the TOF and relative contributions from the two channels are independent of laser pulse energy. In addition, the signal levels for both channels exhibited a linear dependence on laser pulse energy, indicating that both channels result from single photon processes.

In Fig. S3, the TOF spectrum for products detected at $\mathrm{m} / \mathrm{e}=42$, using $8.8 \mathrm{eV}$ photoionization, with the beam source angle set at 10 degrees from the detector axis for photodissociation of dimethylketene at $355.1 \mathrm{~nm}$, is shown. The same translational energy distribution used to simulate the dimethylcarbene $+\mathrm{CO}$ channel detected at $9.9 \mathrm{eV}$, illustrated as Fig. 2 in the main paper, was employed. The observation of dimethylcarbene by photoionization at $8.8 \mathrm{eV}$ is expected based on the vertical ionization energy of $7.7 \mathrm{eV}$, calculated from tabulated enthalpies of formation of the neutral and cationic forms of dimethylcarbene. ${ }^{4}$ Although the propene channel is operative at $355.1 \mathrm{~nm}$, it is not detected using $8.8 \mathrm{eV}$ photoionization because the ionization energy of propene is $9.72 \mathrm{eV}$. As described in the main article, based on the TOF spectra (Figs. 1 and 2) recorded at $355.1 \mathrm{~nm}$, the relative propene yield was found to be $0.16 \pm 0.03$ and that for the dimethylcarbene channel was $0.84 \pm 0.03$. The quoted uncertainties correspond to the range of acceptable simulated fits to the two channels in the $\mathrm{m} / \mathrm{e}=42$ TOF data. In Fig. S4, the TOF spectra are shown for the products from photodissociation of dimethylketene at $340.0 \mathrm{~nm}$. We also recorded TOF spectra at $\mathrm{m} / \mathrm{e}=41$, which were very similar to those shown here at $\mathrm{m} / \mathrm{e}=40$. The relative yield of direct propene increased at $340 \mathrm{~nm}$, with the ratio equal to $0.25 \pm 0.03$ and that for the dimethylcarbene channel equal to $0.75 \pm 0.03$. 
As shown in Fig. S5, excitation at $320.0 \mathrm{~nm}$, led to a further increase in the relative yield for the direct propene channel to $0.32 \pm 0.03$. The data recorded using an excitation wavelength of $371 \mathrm{~nm}$ is shown in Fig. S6. A significant reduction in the contribution to the propene yield, compared to that at $355.1 \mathrm{~nm}$, is evident. From an analysis of the data, the relative propene yield was found to be $0.10 \pm$ 0.03 at $371 \mathrm{~nm}$.

In Fig. S7, we studied the dependence of the signal intensities for both the direct propene $+\mathrm{CO}$ and the dimethylcarbene $+\mathrm{CO}$ product channels as a function of the $355.1 \mathrm{~nm}$ incident laser polarization angle relative to the detector axis. No discernable polarization dependence was observed, indicating that the product angular distributions for the two respective photodissociation channels are both spatially isotropic relative to the electric vector of the incident laser beam. Thus, we report that the anisotropy parameters $(\beta)$ are $0.0+/-0.1$ for the direct propene $+\mathrm{CO}$ channel, as well as for the dimethylcarbene $+\mathrm{CO}$ channel, respectively.

Applying the law of conservation of energy to $\mathrm{CH}_{3} \mathrm{CCH}_{3}+\mathrm{CH}_{3} \mathrm{CHCH}_{2} ; 9.9 \mathrm{eV}$

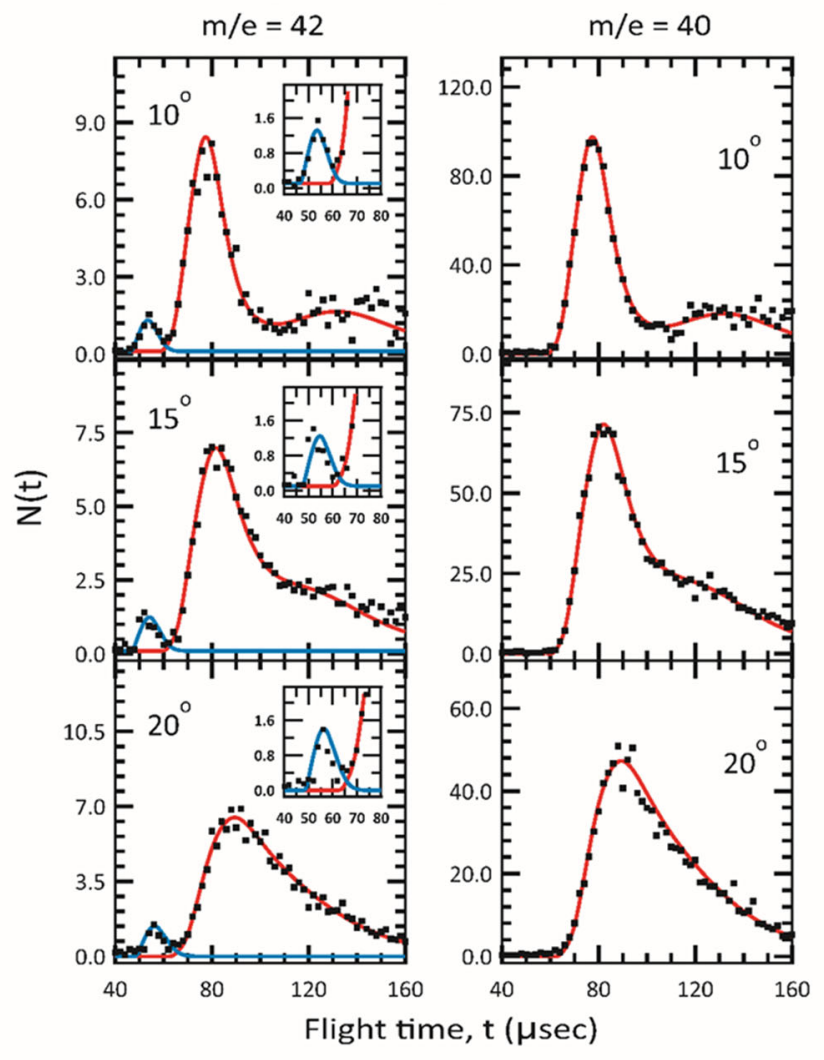

Fig. S4: TOF spectra for products from $340.0 \mathrm{~nm}$ photodissociation of dimethylketene at indicated laboratory angles: black dots are experimental data; red lines are calculated TOF for $\mathrm{CH}_{3} \mathrm{CCH}_{3}+\mathrm{CO}$ channel; blue lines are calculated TOF for propene + $C O$. The relative yield for the propene channel is $0.25 \pm$ 0.03 . The relevant $P(E)$ is depicted in Fig. 5 of the main article. the dissociation of dimethylketene (DMK), the total energy of the excited DMK in excess of the $\mathrm{C}=\mathrm{C}$ bond dissociation energy must be channeled into internal energy (rotational, vibrational and electronic) of the $\mathrm{CH}_{3} \mathrm{CCH}_{3}+\mathrm{CO}$ products, and into their relative product translational energy, $\mathrm{E}_{\text {trans: }}$ :

$$
\mathrm{E}_{\mathrm{int}, \mathrm{DMK}}+\mathrm{E}_{\mathrm{hv}}-\mathrm{D}(\mathrm{DMK})=\mathrm{E}_{\mathrm{int}, \mathrm{CH} 3 \mathrm{CCH} 3}+\mathrm{E}_{\mathrm{int}, \mathrm{CO}}+\mathrm{E}_{\text {trans }}
$$




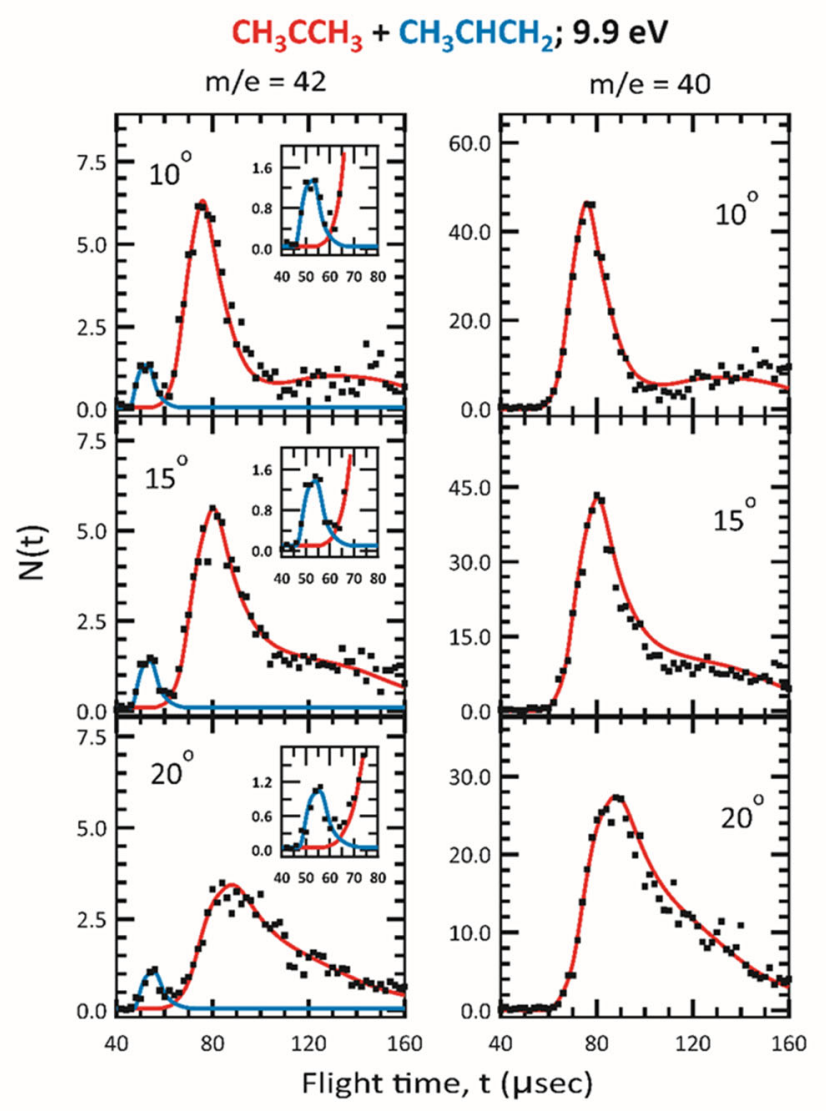

Fig. S5: TOF spectra for products from $320.0 \mathrm{~nm}$ photodissociation of dimethylketene at indicated laboratory angles: black dots are experimental data; red lines are calculated TOF for $\mathrm{CH}_{3} \mathrm{CCH}_{3}+\mathrm{CO}$ channel; blue lines are calculated TOF for propene + $C O$. The relative yield for the propene channel is $0.32 \pm$ 0.03 . The relevant $P(E)$ is depicted in Fig. 5 of the main article.

wavelengths studied here. Therefore, $\mathrm{E}_{\text {int,DMK }} \sim 0$ in Equation S1. The dissociation energy of dimethylketene to produce singlet dimethylcarbene plus $\mathrm{CO}[\mathrm{D}(\mathrm{DMK})]$, requires knowledge of the enthalpies of formation of reactants and products. Because accurate experimental values are not available for dimethylcarbene, we employed the calculated enthalpies of formation (0 K) of $316.2 \pm$ $2.1 \mathrm{~kJ} / \mathrm{mol}$ from the Active Thermochemical Tables. ${ }^{4} \mathrm{We}$ also used the enthalpy of formation for $\mathrm{CO}(0 \mathrm{~K})$ of $-113.803 \pm 0.026 \mathrm{~kJ} / \mathrm{mol}$ from the same
In Equation $\mathrm{S} 1, \mathrm{E}_{\mathrm{int}, \mathrm{DMK}}$ is the initial internal excitation energy of the parent dimethylketene molecules prior to excitation, and $\mathrm{E}_{\mathrm{hv}}$ is the incident photon energy. For a jet-cooled sample of dimethylketene in a molecular beam, the initial rotational temperature is $<10 \mathrm{~K}$, so the initial parent rotational energy is negligible. We observed no evidence for the occurrence of "hot band" activity, i.e., absorption by vibrationally excited dimethylketene molecules at the four excitation

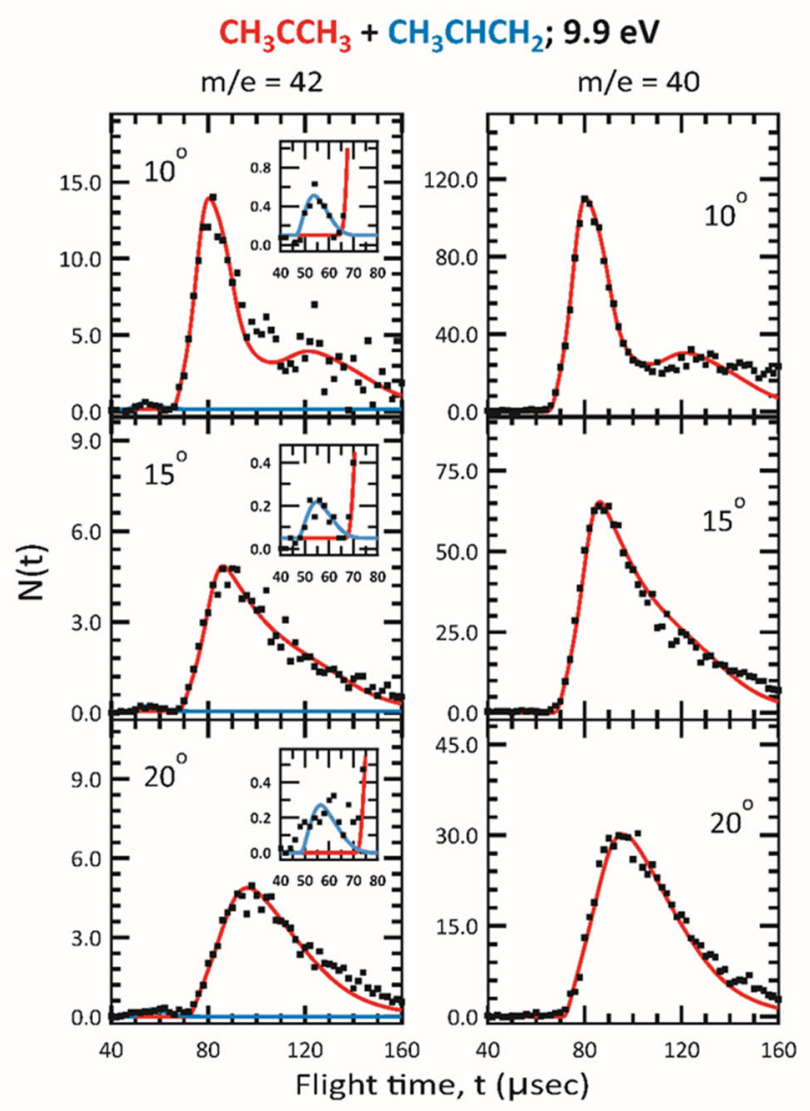

Fig. S6: TOF spectra for products from $371.0 \mathrm{~nm}$ photodissociation of dimethylketene at indicated laboratory angles: black dots are experimental data; red lines are calculated TOF for $\mathrm{CH}_{3} \mathrm{CCH}_{3}+\mathrm{CO}$ channel; blue lines are calculated TOF for propene + $C O$. The relative yield for the propene channel is $0.10 \pm$ 0.03 . The relevant $P(E)$ is depicted in Fig. 5 of the main article. 
source. The experimental enthalpy of formation of dimethylketene is also subject to considerable experimental uncertainty. Therefore, we employed the calculated value $(298 \mathrm{~K})$ of $-89.5 \mathrm{~kJ} / \mathrm{mol}^{5,6}$ Using standard heat capacities published on the NIST website for the elements and dimethylketene, we calculated an enthalpy of formation at $0 \mathrm{~K}$ of $-74 \mathrm{~kJ} / \mathrm{mol}$. Using these standard enthalpies of formation $(0 \mathrm{~K})$, we then calculated the zero Kelvin bond dissociation energy for dimethylketene forming dimethylcarbene $+\mathrm{CO}$ to be $\mathrm{D}(\mathrm{C}=\mathrm{C})=316.2-113.803-(-74.0)=276.4 \mathrm{~kJ} / \mathrm{mol}$. This is nearly identical to the $0 \mathrm{~K}$ value calculated in a recent theoretical study of the photodissociation pathways for methacrolein and methylketene. ${ }^{7}$

Substituting these values into equation $\mathrm{S} 1$, and using $\mathrm{E}_{\mathrm{hv}}=336.75 \mathrm{~kJ} / \mathrm{mol}$ at $355.1 \mathrm{~nm}$,

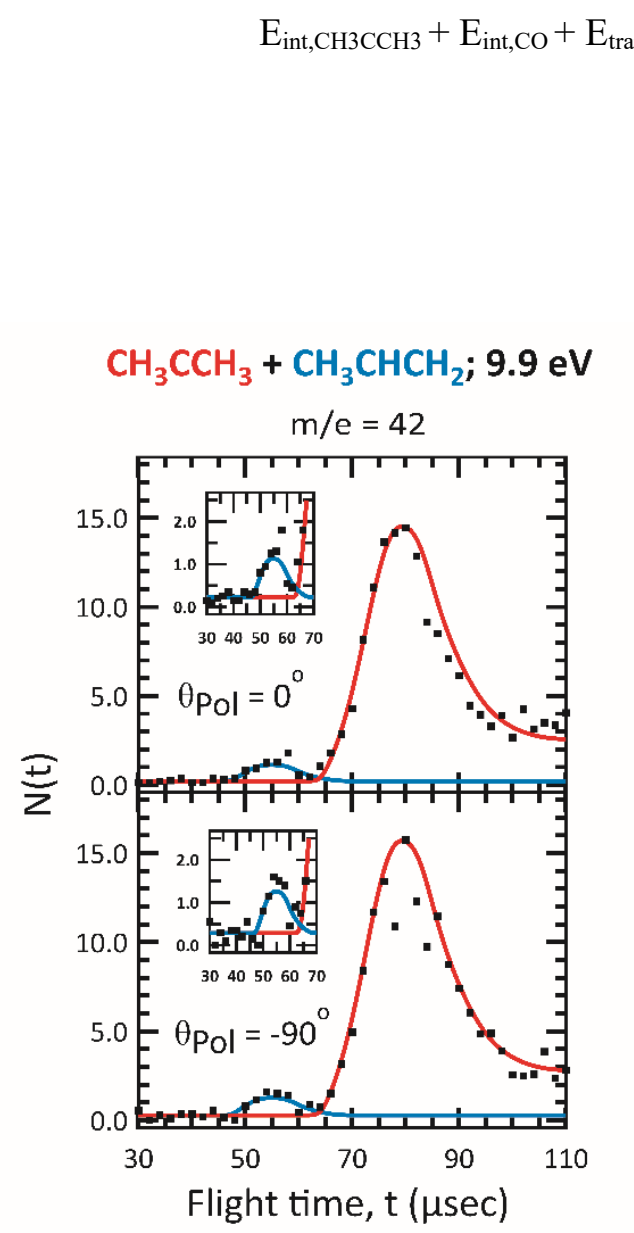

Fig. S7: TOF spectra, at laboratory angle of $10^{\circ}$, for products from $355.1 \mathrm{~nm}$ photodissociation of dimethylketene at indicated polarization angles for the incident photolysis laser beam: black dots are experimental data; red lines are calculated TOF for $\mathrm{CH}_{3} \mathrm{CCH}_{3}+\mathrm{CO}$ channel; blue lines are calculated TOF for propene $+C O$. The anisotropy parameters $(\beta)$ are $0.0+/-0.1$ for both the channels. The relevant $P(E)$ s are depicted in Fig. 2 of the main article.

$$
\begin{aligned}
& =(0+336.75-276.4) \mathrm{kJ} / \mathrm{mol} \\
& =60 \mathrm{~kJ} / \mathrm{mol}
\end{aligned}
$$

The maximum thermodynamically-allowed relative translational energy corresponds to the limiting case where $\mathrm{E}_{\text {int, } \mathrm{CH} 3 \mathrm{CCH} 3}=\mathrm{E}_{\mathrm{int}, \mathrm{CO}}=0$. In this limiting case, $E_{\text {trans, } \max }=60 \mathrm{~kJ} / \mathrm{mol}$. As illustrated in Fig. 3 in the main paper, the observed maximum translational energy was $\sim 46$ $\mathrm{kJ} / \mathrm{mol}$ at $355.1 \mathrm{~nm}$, which is $\sim 14 \mathrm{~kJ} / \mathrm{mol}$ less than the calculated value. Similarly, as illustrated in Fig. 5, we consistently observed maximum translational energies that were smaller than the calculated maximum values for each wavelength. This behavior contrasts that seen for methylketene producing methylcarbene $+\mathrm{CO}$, where the maximum observed translational energy release values were in very close agreement to the calculated values. ${ }^{1}$ For dimethylketene photodissociation, formation of dimethylcarbene $+\mathrm{CO}$ involves simple bond fission on $\mathrm{S}_{0}$ and no potential energy barrier in excess of the bond dissociation energy is anticipated. Therefore, a significant fraction of 
available energy is channeled into vibrational energy of the dimethylcarbene and the maximum theoretical translational energy release (corresponding to zero internal excitation of products) may not be realized. In the photodissociation of methylketene, on the other hand, products are formed with a significantly relative translational energy release because dissociation proceeds on the triplet PES over a potential energy barrier ${ }^{1}$

\section{References:}

1. Datta, S.; Davis, H.F. Direct Observation of Ethylidene, the Elusive High-Energy Isomer of Ethylene. J. Phys. Chem. Lett. 2020, 11, 10476-10481.

2. Albert D.R.; Davis H.F. Experimental Studies of Bimolecular Reaction Dynamics Using Pulsed Tabletop Vacuum Ultraviolet Photoionization Detection, Phys. Chem. Chem. Phys. 2013, 15, 14566-14580.

3. Albert, D.R.; Proctor, D.L.; Davis, H.F. High-intensity coherent vacuum ultraviolet source using unfocussed commercial dye lasers. Rev. Sci. Instrum. 2013, 84, 063104.

4. Ruscic, B.; Bross, D.H. Active Thermochemical Tables (ATcT), version $1.122 \mathrm{~g}$ of the Thermochemical Network; Argonne National Laboratory: Lemont, IL, 2019. www.ATcT.anl.gov (accessed March 22, 2021).

5. Simmie, J.M.; Metcalfe, W.K.; Curran, H.J. Ketene Thermochemistry. Chem. Phys. Chem. 2008, 9, 700-702.

6. Sumathi, R.; Green, W.H. Thermodynamic Properties of Ketenes: Group Additivity Values from Quantum Chemical Calculations. J. Phys. Chem. A 2002, 106, 7937- 7949.

7. So, S.; Wille, U.; DaSilva, G. Photoisomerization of Methyl Vinyl Ketone and Methacrolein in the Troposphere: A Theoretical Investigation of Ground-State Reaction Pathways. ACS Earth Space Chem. 2018, 2, 753-763. 